\title{
Intracultural Variation in Cross-Cultural Gerontology
}

\author{
Robert W. Schrauf
}

Published online: 1 May 2009

(C) Springer Science + Business Media, LLC 2009

Intracultural variation refers to specifically cultural differences between individuals, all of whom belong to the same, larger cultural group. Given shared cultural beliefs and norms, individuals will nevertheless differ in their adherence to these beliefs and norms. For example, Americans may generally know and value participatory democracy and the right to vote, but Americans differ in how well they understand the process or participate in it. In a second sense, intracultural variation refers to the existence of sub-cultural groupings within a larger cultural group. For instance, as regards the latter, researchers interested in multicultural phenomena in the United States often use the broad ethnic categories devised by Office of Budget and Management (Federal Register, October 30, 1997) and employed in Census 2000 to distinguish among ethnic groups. These include five minimum categories for data on race (American Indian or Alaska Native, Asian, Black or African American, Native Hawaiian or Other Pacific Islander, and White), plus two categories for data on ethnicity ("Hispanic or Latino" and "Not Hispanic or Latino"). Nevertheless, the research community is increasingly aware that important distinctions must be made within these broad categories. For instance, "Hispanic" or "Asian" include multiple distinct national and local cultures. "Black or African American" collapses over differences between African Americans who are descendents of slaves in the U.S. and African or West Indian immigrants.

The notion of intracultural variation is related to, but different from, the notion of individual differences. The study of individual differences involves identifying and measuring within-group patterns of variation in independent and dependent variables, but such differences need not be explicitly cultural, nor do they necessarily involve the identification and characterization of subgroups. For instance, at the level of the group, one immigrant community may differ from another in levels of acquisition of English, but inspection of individual differences in formal education, access to resources, and frequency of visits to the home country may well explain these differences. These essentially psychosocial variables may be powerful predictors of English proficiency, but because they do not involve specifically cultural ideation, cultural value sets, or culturally-shaped

R. W. Schrauf $(\bowtie)$

Pennsylvania State University, University Park, PA, USA

e-mail: rws23@psu.edu 
affective configurations, they are examples of individual differences and not intracultural variation. Were individuals within the groups to espouse different sets of beliefs about the value and meanings of English, this would be an example of intracultural variation.

In general, inter-and intra-cultural variation has been the concern of anthropologists, whereas talk of between-and within-group designs and the individual differences approach evoke a more general social scientific paradigm. Since cross-cultural research has become a focus in many social science disciplines, it may be useful to trace some of the development of these concepts in the two root disciplines in which much of the theoretical and methodological development has take place: cultural anthropology and cross-cultural psychology. Where possible, references to research in cross-cultural gerontology will be made.

\section{Intracultural Variation in Anthropology}

In cultural anthropology, cross-cultural research strictly so-called, involves "systematic comparisons across cultures - comparisons that, we expect, will answer questions about the incidence, distribution, and causes of cultural variation" (Ember and Ember 1998, p. 648). In effect, cultures or societies are the unit of analysis, and the researcher is interested in investigating between-and within-culture variation of one or more traits. Such research is driven by some combination of the following aims: (a) description-looking at the prevalence or frequency of a trait (or traits) across cultures, (b) explanation-identifying the causes of a trait, (c) implications - characterizing the consequences of particular traits for the societies involved, and (d) relational-identifying and weighing the mutual influence of cultural traits (Ember and Ember 1998, p. 650). The comparisons usually involve whole societies or cultural groups within a multicultural society. As an example of the former in aging research, Shalinsky and Glascock (1988) used ethnographic evidence from 55 nonindustrial societies in the Human Relations Area Files (M. Ember 1997) to examine death-hastening practices targeting infants and the elderly. Such practices were more frequent among societies that placed infants and/or older adults in liminal categories as presocial or post-social. More relevant to specifically intracultural variation, Schrauf (1999) identified factors predicting 'mother-tongue' maintenance among 11 ethnic groups in North America and found that residential patterns and religious practices predicted first-language maintenance into the third generation. Nevertheless, in the cross-cultural research framework in anthropology, the focus is on intercultural variation and less so on intracultural variation.

The underlying data for such studies is ethnographic and based on fieldwork-either new data gathered in primary fieldwork or existing data in the published ethnographic record (e.g. the Human Relations Area Files). Ethnography is of course the foundational method in anthropology, and given that ethnography is based on systematic participant observation sustained over a period of time, ethnographies commonly document intracultural variation among the peoples studied. First, at the level of daily fieldwork, it is usually patently obvious that culture members differ in their uptake and expression of cultural practices and beliefs. Second, once written up, ethnographies are rhetorically persuasive precisely because they hold to the light the striking diversity-within-unity of the societies that they portray ("so different, yet just like us"). An excellent example in gerontology is Traphagan's (2000) investigation of aging in Japan, through the eyes of the elderly in the town of Kanegasaki in Iwate Prefecture. Thus, ethnographies are relatively strong in their presentation of intracultural variation, but in contrast to cross-cultural research properly-so-called, they often leave intercultural comparisons fairly implicit. 
In more recent ethnographic practice, anthropologists have attempted to systematically interrelate levels of sociocultural analysis to show how micro-contexts are intimately connected to, and shaped by global macro-contexts (Lewellen 2002; Marcus 1998). Here, observations about intracultural variation are recruited into the intercultural comparative project at higher national, regional, and global levels of analysis. To cite one example from the aging literature, Lock (1995) shows that the medicalization of female menopause in North American biomedicine does not articulate easily with the cultural logic of menopause as experienced by women in Japan. Once placed in a cross-cultural comparative framework, the intracultural variation in the Japanese context nuances the assumptions made by a putatively universal biomedicine.

Finally, in a quite focused sense, cognitive anthropologists have developed a formal mathematical model of intracultural variation as cultural consensus theory. Consensus theory recognizes that, even though all individuals from the same culture probably do agree with one another about common beliefs, attitudes, values, schemata, and scripts, their agreement is far from absolute. Being human, they vary. Further, the differences between individuals in their knowledge of cultural beliefs, attitudes, values, schemata, and scripts can be measured. In essence, given an overall pattern of agreement ('one culture') there will always be differences between people (varying 'cultural competence') within the larger pattern of agreement and possibly consistent patterns of agreement among subsets of individuals ('subcultures'). In gerontology, Barg and colleagues (2006) used cultural consensus analysis to examine intracultural variation in depressive symptoms among older African Americans in the U.S. and found high levels of interindividual agreement about the symptoms and meanings of depression. In particular, they found that loneliness was a key feature of depression for their participants, despite the fact that loneliness is not a diagnostic feature of depression. Schrauf and Sanchez (2008) have described how the method may be used to assess subtle cultural differences between young and old in their ideation about everyday cultural domains (e.g. emotions, illnesses, gender).

\section{Individual Differences in Cross-Cultural Social Science}

Moving from anthropology to the larger arena of the social sciences, we find again a range of theory and methods concerning cross-cultural comparisons. However, the underlying methodological paradigm is largely consistent across disciplines. At the risk of oversimplification, cross-cultural psychology may be taken as representative of these approaches. The goals of cross-cultural psychology are to understand the range of variation of psychological variables across cultural contexts and, in many cases, to search for and characterize psychological universals in human experience and functioning (Adamopoulos and Lonner 2001). This fundamentally quantitative paradigm is driven by the logicoempirical scientific method, and psychological constructs are operationalized as observable, measureable variables. Herein, of course, lies the challenge. Establishing the cross-cultural validity of data collection and analytic techniques is absolutely necessary to make meaningful comparisons. In this vein, the field has made considerable methodological progress and set the standard for much cross-cultural empirical research (e.g. F. Van de Vijver 2001). Issues of sampling, linguistic translation and adaptation of instruments, and the achievement of construct equivalence are key elements in credible cross-cultural research design (e.g. Hambleton et al. 2005; Fons van de Vijver and Leung 1997). In this tradition, it is more common to speak of individual differences (psychological and social factors) as described above, rather than intracultural variation with its characteristic emphasis on cultural beliefs and norms. 
In gerontology, Whitfield and colleagues have been instrumental in articulating the importance of individual differences research when making ethnic comparisons in a multicultural society (Whitfield and Baker-Thomas 1999; Whitfield et al. 2008). Their research points again to the necessity of ensuring measurement and construct equivalence across groups in order to make meaningful comparisons, but they also draw attention to critical sampling issues and within-group heterogeneity. In particular, they note that betweengroup comparisons require sufficient numbers of participants in all samples to have sufficient power to detect differences, and all groups must have similar variances on the dependent variable (a key assumption in the ANOVA model). Relative to individual differences, Whitfield et al. (2008) stress the important role of within-group designs that adequately survey within-group heterogeneity on psychosocial variables that potentially affect outcome variables. As noted above, in this approach, individual differences research focuses on variation between individuals that might be masked by group means, and this differs from intracultural variation in being focused on factors not explicitly glossed as cultural.

\section{The Papers in This Special Issue}

The papers in this volume cut across both the anthropological tradition of intracultural variation and the social science emphasis on individual differences, but the emphasis is on the former. Each contribution focuses especially on intracultural variation and the role of specifically cultural factors in within-group heterogeneity.

Language and bilingualism in particular are explored as a form of intracultural variation among ethnic elders in papers by Nicole Mueller and Robert Schrauf. Both papers remind us that bilinguals are in fact biculturals to one extent or another, and hence the contexts and frequency of use of the two (or more) languages become important indicators of cultural stance and cultural competence. Mueller's ethnographic research takes her into a Louisiana nursing home where "the last generation of French speakers who grew up with French as the primary language in their homes is alive today." Whereas the majority of residents in this facility are in fact French-English bilinguals, the default language is English. Where French is used, it references the oral home culture of childhood and youth associated with limited financial resources and limited educational opportunity. Nevertheless, as a language of domestic intimacies, it is a mark of identity for these residents, despite their compliance with the monocultural and monolingual English environment of the facility.

Schrauf addresses the English language proficiency and patterns of English language-use among older Puerto Rican, English-Spanish bilinguals who live in a Spanish-speaking, ethnic neighborhood in a large urban area. In particular, using a formal test of English proficiency, Schrauf divided these participants into three proficiency groups and inspected their self-reported patterns of social, conversational English and use-of-English in mental practices and inner speech. The group with lowest English proficiency patterned quite differently from both the fluent and intermediate proficiency groups both in terms of antecedents (higher age-at-immigration) and consequences (lower current SES, less likely to own a home vs. rent, and lower levels of acculturation to US society). Here, language proficiency in English sets off one group of older Puerto Ricans from the rest of the sample on a number of psychosocial and cultural factors and in effect creates a subcultural group within the larger ethnic population. Language proficiency correlates with lower levels of acculturation (beliefs and practices) and is a cause and marker of intracultural variation.

Jang, Chiriboga, Herrera, and Branch examine intracultural variation among Cubans living in Havana vs. Cubans living in Miami on self-reported health. In essence, this study 
looks at one culture (Cuban) whose members are dispersed across two spaces - one in the home country, one in the diaspora-which sets the stage for examining intracultural variation. Interestingly, the study found no difference between sites in percentage reporting poor health (13\%). This is all the more striking given the critical differences between sites: those in Miami reported higher education levels and far fewer in Miami reported financial strain $(20 \%)$ than Havana (78\%). Groups did differ, however, in the association between reported chronic conditions and reported poor health: whereas these were related for those in Havana, the relationship did not hold in Miami, perhaps because those in Miami felt that such conditions were more manageable. In the end, however, groups did not differ in the outcome variable, and what differences were found reflect more individual differences (e.g. financial strain, education) than differences in understandings of, or adherence to, cultural beliefs and norms (intracultural variation).

The paper by Fiori, Consedine, and Magai most directly reflects intracultural variation as sub-groupings within larger ethnic groupings (as described in the introduction to this article). This is a study of late life attachment styles among seven ethnic groups in the United States that represent shades of variation where "lumping" usually occurs. Instead of Hispanics, there are samples of Dominicans and Puerto Ricans. Instead of Blacks, there are samples of Haitians, African-Americans, and English-speaking Caribbeans. Instead of Whites, there are samples of Eastern Europeans and European Americans. Although a 'dismissive avoidance' attachment style was found for the majority of older adults (consistent with the literature), groups differed in their emphases on the remaining styles (secure, fearful avoidance, preoccupation).

The paper by Sims, Allaire, Gamaldo, Edwards, and Whitfield examines the dedifferentiation hypothesis among African Americans in the United States. Briefly, this is the hypothesis that cognitive abilities that emerge and integrate during childhood undergo differentiation in late adolescence, remain stable through adulthood, and then begin a process of de-differentiation in old age. Since African Americans often have poorer health outcomes and fewer years of education than whites, and since these differences may cumulatively affect cognitive functioning in late life, Sims et al. conducted a within-group study to assess whether dedifferentiation occurs among African American elders. They find little evidence for the dedifferentiation hypothesis in their African American sample of 512 adults, divided into middle aged, young-old, and old-old. The significance of the paper lies in "building dedifferentiation findings for African-American elders, a group with unique cultural experiences that has previously been excluded from studies of dedifferentiation," but the authors suggest that the exploration of other covariates "such as health, education, social support, religious practices, SES, and other unique cultural factors that may affect cognitive performance." Future studies that address health status, education, and social support would be examples of individual differences research. Studies addressing religious practices and "other unique cultural factors" would focus on intracultural variation.

As this brief introduction and a careful reading of these papers should make clear, there is considerable need in cross-cultural gerontology for attention to within-group heterogeneity and specifically to both the psychosocial factors (individual differences) and the cultural factors (intracultural variation) that comprise this heterogeneity. In current research, investigators are probably more attuned to psychological than cultural factors, perhaps in part because much gerontological research focuses on aging as an individual micro-level phenomenon to the neglect of mesolevels (communities and social organizations) and macro-levels (society) (Hagestad and Dannefer 2001). However, to claim a real cross-cultural difference requires some attention to specifically cultural factors and intracultural variation around these factors. Perhaps these thoughts and these papers will help refine the cross-cultural project. 


\section{References}

Adamopoulos, J., \& Lonner, W. J. (2001). Culture and psychology at a crossroad: Historical perspective and theoretical analysis. In D. Matsumoto (Ed.), The handbook of culture and psychology, pp. 11-50. New York: Oxford University Press.

Barg, F. K., Huss-Ashmore, R., Wittink, M. N., Murray, G. F., Bogner, H. R., \& Gallo, J. J. (2006). A mixedmethods approach to understanding loneliness and depression in older adults. Journals of Gerontology. Series B, Psychological Sciences and Social Sciences, 61, S329-S339.

Ember, C. R., \& Ember, M. (1998). Cross-cultural research. In H. R. Bernard (Ed.), Handbook of methods in cultural anthropology, pp. 647-687. Walnut Creek, CA: Altamira.

Ember, M. (1997). Evolution of the human relations area files. Cross-Cultural Research, 31, 3-15.

Hagestad, G. O., \& Dannefer, D. (2001). Concepts and theories of aging: Beyond microfication in social science approaches. In R. H. Binstock \& L. K. George (Eds.), Handbook of aging and the social sciences, pp. 3-21. New York: Academic.

Hambleton, R. K., Merenda, P. F., \& Spielberger, C. D. (eds). (2005). Adapting educational and psychological tests for cross-cultural assessment. Mahwah, New Jersey: LEA.

Lewellen, T. C. (2002). The anthropology of globalization: Cultural anthropology enters the 21st century. Westport, Connecticut: Bergin \& Garvey.

Lock, M. M. (1995). Encounters with aging: Mythologies of menopause in Japan and North America. Berkeley, CA: University of California Press.

Marcus, G. E. (1998). Ethnography through thick and thin. Princeton, New Jersey: Princeton University Press.

Schrauf, R. W. (1999). Mother tongue maintenance among north american ethnic groups. Cross-Cultural Research, 33(2), 175-192.

Schrauf, R. W., \& Sanchez, J. (2008). Using freelisting to identify, assess, and characterize age differences in shared cultural domains. Journal of Gerontology: Social Sciences, 63B(6), S385-S393.

Shalinsky, A., \& Glascock, A. (1988). Killing infants and the aged in nonindustrial societies: Removing the liminal. Social Science Journal, 25(3), 277-287.

Traphagan, J. W. (2000). Taming oblivion: Aging bodies and the fear of senility in Japan. Albany, New York: State University of New York Press.

Van de Vijver, F. (2001). The evolution of cross-cultural research methods. In D. Matsumoto (Ed.), The handbook of culture and psychology, pp. 77-97. New York: Oxford.

van de Vijver, F., \& Leung, K. (1997). Methods and data analysis for cross-cultural research. Thousand Oaks, CA: Sage.

Whitfield, K. E., \& Baker-Thomas, T. (1999). Individual differences in aging minorities. International Journal of Aging and Human Development, 48, 73-79.

Whitfield, K. E., Allaire, J. C., Belue, R., \& Edwards, C. L. (2008). Are comparisons the answer to understanding behavioral aspects of aging in racial and ethnic groups? Journal of Gerontology: Social Sciences, 63B(5), 301-P308. 